\title{
Evidence-based mental health care and implementation science in low- and middle-income countries
}

\author{
G. Thornicroft* \\ Health Service and Population Research Department, King's College London, Institute of Psychiatry, De Crespigny Park, London, England
}

\begin{abstract}
Although the evidence base for what to do about the mental health gap in low- and middle-income countries (LAMICs) has improved significantly over the last decade, mental health care in LAMICs still provide services to only a small minority of people with mental disorders. The problem is how to translate the relevant body of scientific knowledge into routine practice. It is clear from over two decades of research that the creation of evidence-based guidelines is necessary but not sufficient for evidence-based practice, whether in high- or low-income settings. In this Editorial, I discuss whether the recent development of 'implementation science' may offer an opportunity towards effective guideline implementation in low- and medium-income settings, so that clinical practice is more often based on evidence that does lead to patient benefit.
\end{abstract}

First published online 28 May 2012

Key words: Guidelines, implementation, low- and middle-income countries.

If there can be 'no health without mental health' (Prince et al. 2007), then a health system does not function properly if it cannot protect and take care of the basic health rights and needs of people who are unwell or vulnerable - including people with mental illness (Chisholm et al. 2007). In most low- and middleincome countries (LAMICs), resources and services for mental health are meagre in the extreme, with lowincome countries allocating on average $0.5 \%$, and lower-middle income countries $1.9 \%$ of their health budget to the treatment and the prevention of mental disorders, even though they represent over $10 \%$ of the overall disease burden (Saxena et al. 2007; World Health Organization, 2011). In LAMICs, there is on average one psychiatrist per 1.7 million inhabitants and one psychiatric inpatient bed per 42000 inhabitants (Jacob et al. 2007). Most of the funds that are made available by governments are directed towards the running costs of mental hospital service provision. This limits the development of more equitable and cost-effective community-based services. The result of inadequate, inequitable and inefficient resourcing for mental health is a substantial treatment gap (Thornicroft, 2007). An international survey supported by WHO showed that $76-85 \%$ of people with severe

*Address for correspondence: Professor Graham Thornicroft, Professor of Community Psychiatry, Health Service and Population Research Department, King's College London, Institute of Psychiatry, De Crespigny Park, London SE5 8AF, England.

(Email:graham.thornicroft@kcl.ac.uk) mental disorders in low-income countries had not received any treatment in the previous 12 months (Demyttenaere et al. 2004). The adverse consequences of this unmet need include the violation or abuse of human rights (Callard et al. 2012), long-term disability and ill-health (Chisholm et al. 2005), and increased mortality (Thornicroft, 2011; Wahlbeck et al. 2011).

It is therefore clear that the quantity and quality of mental health care in LAMICs are grossly deficient. Is there sufficient relevant evidence from LAMICs on cost-effective interventions that do need to be put into practice? To date much of the mental health research undertaken in LAMICs has been on classification, epidemiology and identification of mental disorders. Yet recent developments include improved policy guidance, treatment guidelines and generation of evidence on the cost-effectiveness of interventions in LAMICs, especially those relevant for primary and community care staff (Patel \& Thornicroft, 2009). Accordingly, the knowledge base for what to do about the escalating burden of mental disorders has improved significantly over the last decade (World Economic Forum, 2011). Landmark developments include the World Health Report in 2001 (World Health Organization, 2001), two Lancet series on global mental health in 2007 and 2011, the establishment of a Global Movement for Mental Health, the development of WHO's mhGAP programme for scaling up services for mental, neurological and substance use disorders (complete with its evidence-based intervention guide) (Barbui et al. 2010; World Health 


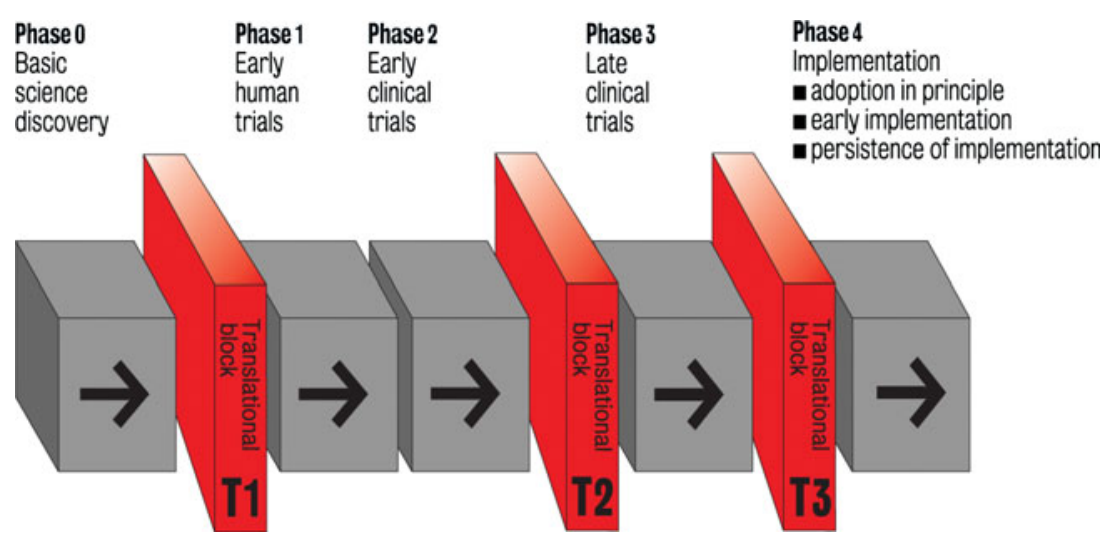

Fig. 1. Five phases and three blocks in the translational medicine continuum. (Thornicroft et al., 2011)

Organization, 2010; Dua et al. 2011), and the recent Grand Challenges in Global Mental Health review (Collins et al. 2011), which singled out improved treatment and access to care as the core agenda for future research and development efforts. There is therefore now a strong and growing international consensus that the shortage of specialist mental health care staff requires a policy shift to a clear commitment that LAMICs horizontally integrate mental health care into primary care and into maternal health care (Eaton et al. 2011; United Nations, 2011). Such integration now also provides opportunities for reducing the stigma of mental illness, which in itself is a major barrier to accessing care and to social inclusion (Thornicroft et al. 2009).

The problem is how to translate this body of knowledge into practice. At its root these are health systems issues. Past failures have been partly due to a shortage of political will and funding, and also reflect a technical set of challenges around how best to address health system issues and constraints as they relate to integrated mental health service provision in nonspecialized settings, including human resources and capacity building, information systems, health financing and service delivery (Saraceno et al. 2007).

The recently developed World Health Organization mhGAP Intervention Guide therefore offers a step change opportunity to practitioners in LAMICs with its practical guidance on the identification and treatment of mental disorders in primary and community care settings. The fundamental public health questions are: (i) can such guidelines be put into practice in routine clinical settings, (ii) if so, do they confer patient benefit and (iii) does their use contribute to an increase in the treated prevalence rate for specified conditions?

It is clear from over two decades of research that the creation of guidelines is necessary but not sufficient for evidence-based practice, whether in high- or lowincome settings. As a consequence there has been the recent rapid development of 'implementation science' (Madon et al. 2007; Eccles et al. 2009), with some applications in the field of mental health (Bauer, 2002; Michie et al. 2007; Evans-Lacko et al. 2010). A recent review has summarized the factors that have been identified as facilitators or barriers to the implementation of mental illness-related clinical guidelines (Tansella \& Thornicroft, 2009) and distinguished between: (i) the adoption in principle of guidelines; (ii) early implementation and (iii) their sustained use over time, namely actions at Phase 4 as shown in Fig. 1 (Thornicroft et al. 2011).

The two critical needs are clear. First, well designed, sufficiently statistically powered, properly conducted, and openly reported intervention studies (including randomized controlled trials) of interventions (at both the patient/practitioner and at the clinical team/ facility level), which are utterly realistic and practical in low- and middle-income settings. This requires immediate investment from research funding councils and donors in high-income countries. When this evidence base is in place then mental health service provision in LAMICS can be based on information that is fit for the purpose. Until then using the limited information that has been directly derived from LAMCs, along with the unsatisfactory proxy of adapting evidence from high-income settings, is the least bad basis for action. Second, there a requirement to understand more clearly how and why guidelines can be implemented in low-and medium-income settings so that clinical practice is more often based on relevant evidence that does lead to patient benefit.

\section{Acknowledgements}

G.T. is funded in relation to a National Institute for Health Research (NIHR) Applied Programme grant awarded to the South London and Maudsley NHS 
Foundation Trust (GT), and in relation to the NIHR Specialist Mental Health Biomedical Research Centre at the Institute of Psychiatry, King's College London and the South London and Maudsley NHS Foundation Trust. All opinions expressed here are solely those of the author. The author would like to acknowledge the contribution of Professor Michele Tansella towards this approach to implementation science.

\section{Conflicts of Interest}

None.

\section{References}

Barbui C, Dua T, Van Ommeren M, Yasamy MT, Fleischmann A, Clark N et al. (2010). Challenges in Developing Evidence-Based Recommendations Using the GRADE Approach: The Case of Mental, Neurological, and Substance Use Disorders. PLoS Medicine 7(8):e1000322.

Bauer MS (2002). A review of quantitative studies of adherence to mental health clinical practice guidelines. Harvard Review of Psychiatry 10, 138-153.

Callard F, Sartorius N, Arboleda-Florez J, Bartlett P, Helmchen H, Stuart H, Taborda J, Thornicroft G (2012). Mental Illness, Discrimination and the Law: Fighting for Social Justice. Wiley Blackwell, London.

Chisholm D, Flisher AJ, Lund C, Patel V, Saxena S, Thornicroft G, Tomlinson M (2007). Scale up services for mental disorders: a call for action. Lancet 370, 1241-1252.

Chisholm D, Van Ommeren M, Ayuso-Mateos JL, Saxena S (2005). Cost-effectiveness of clinical interventions for reducing the global burden of bipolar disorder. British Journal of Psychiatry 187, 559-567.

Collins PY, Patel V, Joestl SS, March D, Insel TR, Daar AS, Anderson W, Dhansay MA, Phillips A, Shurin S, Walport M, Ewart W, Savill SJ, Bordin IA, Costello EJ, Durkin M, Fairburn C, Glass RI, Hall W, Huang Y, Hyman SE, Jamison K, Kaaya S, Kapur S, Kleinman A, Ogunniyi A, Otero-Ojeda A, Poo MM, Ravindranath V, Sahakian BJ, Saxena S, Singer PA, Stein DJ (2011). Grand challenges in global mental health. Nature 475, 27-30.

Demyttenaere K, Bruffaerts R, Posada-Villa J, Gasquet I, Kovess V, Lepine JP, Angermeyer MC, Bernert S, de Girolamo G, Morosini P, Polidori G, Kikkawa T, Kawakami N, Ono Y, Takeshima T, Uda H, Karam EG, Fayyad JA, Karam AN, Mneimneh ZN, Medina-Mora ME, Borges G, Lara C, Ormel J, Gureje O, Shen Y, Huang Y, Zhang M, Alonso J, Haro JM, Vilagut G, Bromet EJ, Gluzman S, Webb C, Kessler RC, Merikangas KR, Anthony JC, Von Korff MR, Wang PS, Brugha TS, Guilar-Gaxiola S, Lee S, Heeringa S, Pennell BE, Zaslavsky AM, Ustun TB, Chatterji S (2004). Prevalence, severity, and unmet need for treatment of mental disorders in the World Health Organization World Mental Health Surveys. Journal of American Medical Association 291, 2581-2590.
Dua T, Barbui C, Clark N, Fleischmann A, van Ommeren M, Poznyak V, Yasamy MT, Thornicroft G, Saxena S (2011). Evidence based guidelines for mental, neurological and substance use disorders in low- and middle-income countries: summary of $\mathrm{WHO}$ recommendations. PLoS Medicine 8, 1-11.

Eaton J, McCay L, Semrau M, Chatterjee S, Baingana F, Araya R, Ntulo C, Thornicroft G, Saxena S (2011). Scale up of services for mental health in low-income and middle-income countries. Lancet 378, 1592-1603.

Eccles MP, Armstrong D, Baker R, Cleary K, Davies H, Davies S, Glasziou P, Ilott I, Kinmonth AL, Leng G, Logan S, Marteau T, Michie S, Rogers H, Rycroft-Malone J, Sibbald B (2009). An implementation research agenda. Implementation Science 4, 18.

Evans-Lacko S, Jarrett M, McCrone P, Thornicroft G (2010). Facilitators and barriers to implementing clinical care pathways. BMC Health Service Research 10, 182.

Jacob KS, Sharan P, Mirza I, Garrido-Cumbrera M, Seedat S, Mari JJ, Sreenivas V, Saxena S (2007). Mental health systems in countries: where are we now? Lancet 370, 10611077.

Madon T, Hofman KJ, Kupfer L, Glass RI (2007). Public health. Implementation science. Science 318, 1728-1729.

Michie S, Pilling S, Garety P, Whitty P, Eccles MP, Johnston M, Simmons J (2007). Difficulties implementing a mental health guideline: an exploratory investigation using psychological theory. Implementation Science 2, 8.

Patel V, Thornicroft G (2009). Packages of care for mental, neurological, and substance use disorders in low- and middle-income countries: PLoS Medicine Series. PLoS Medicine 6, 10.

Prince M, Patel V, Saxena S, Maj M, Maselko J, Phillips MR, Rahman A (2007). No health without mental health. Lancet 370, 859-877.

Saraceno B, van Ommeren M, Batniji R, Cohen A, Gureje O, Mahoney J, Sridhar D, Underhill C (2007). Barriers to improvement of mental health services in low-income and middle-income countries. Lancet 370, 1164-1174.

Saxena S, Thornicroft G, Knapp M, Whiteford H (2007). Resources for mental health: scarcity, inequity, and inefficiency. Lancet 370, 878-889.

Tansella M, Thornicroft G (2009). Implementation science: understanding the translation of evidence into practice. British Journal of Psychiatry 195, 283-285.

Thornicroft G (2007). Most people with mental illness are not treated. Lancet 370, 807-808.

Thornicroft G (2011). Physical health disparities and mental illness: the scandal of premature mortality. British Journal of Psychiatry 199, 441-442.

Thornicroft G, Brohan E, Rose D, Sartorius N, Leese M (2009). Global pattern of experienced and anticipated discrimination against people with schizophrenia: a cross-sectional survey. Lancet 373, 408-415.

Thornicroft G, Lempp H, Tansella M (2011). The place of implementation science in the translational medicine continuum. Psychological Medicine 41, 2015-2021.

United Nations (2011). United Nations General Assembly Special Session on Non-Communicable Diseases: Political Declaration of 
the High-Level Meeting of the General Assembly on the Prevention and Control of Non-communicable Diseases (document A/66/L.1). United Nations: New York.

Wahlbeck K, Westman J, Nordentoft M, Gissler M, Laursen TM (2011). Outcomes of Nordic mental health systems: Life expectancy of patients with mental disorders. British Journal of Psychiatry 199, 453-458.

World Economic Forum (2011). The Global Economic Burden of Non-Communicable Diseases. World Economic Forum: Geneva.
World Health Organization (2001). World Health Report 2001. Mental Health: New Understanding, New Hope. World Health Organization: Geneva.

World Health Organization (2010). mhGAP Intervention Guide for Mental, Neurological and Substance Use Disorders in Non-Specialized Health Settings: Mental Health Gap Action Programme (mhGAP). World Health Organization: Geneva.

World Health Organization (2011). WHO Mental Health Atlas. World Health Organization: Geneva. 